\title{
ISOLAMENTO DE DERMATÓFITOS DO PELO DE CÃES E GATOS PERTENCENTES A PROPRIETÁRIOS COM DIAGNÓSTICO DE DERMATOFITOSE
}

\author{
Daniele Bier 1 , Marconi Rodrigues de Farias², Marisol Dominguez Muro', Luciana \\ Maria Faiad Soni ${ }^{3}$, Vânia Oliveira Carvalho', Cláudia Turra Pimpão² \\ 1 UFPR \\ 2 PUC-PR \\ 3 Mycolab - Laboratório de Micologia \\ Correspondência: Daniele Bier: danielebier@ufpr.br
}

RESUMO: Este estudo avaliou o potencial de cães e gatos em disseminar fungos dermatófitos, o perfil epidemiológico mais suscetível e as principais síndromes clínicas associados à dermatofitose humana. Foram coletados e cultivados pelos e escamas de 21 gatos e 33 cães domiciliados com 25 proprietários com diagnóstico de dermatofitose. Tanto crianças como adultos adquiriram a dermatofitose a partir do contato com cães e gatos. A maioria das crianças apresentava Tinea capitis enquanto os adultos apresentavam Tinea corporis. Dos animais avaliados, 67\% (36/54) foram positivos para fungos dermatófitos, sendo $95 \%$ (20/21) dos gatos e $49 \%$ (16/33) dos cães positivos. Dos gatos analisados, tanto assintomáticos como sintomáticos podem ter transmitido a doença. Quanto aos cães, os assintomáticos foram provavelmente os principais veiculadores da dermatofitose aos seus proprietários. Das amostras positivas em gatos, e em $94,4 \%$ das positivas em cães, o dermatófito isolado foi o Microsporum canis.

Palavras-chave: adultos; crianças; Microsporum; Tinea

\section{ISOLATION OF DERMATOPHYTES FROM THE HAIR COAT OF PET DOGS AND CATS BELONGING TO OWNERS DIAGNOSED WITH DERMATOPHYTOSIS}

\begin{abstract}
This study evaluated the potential of dogs and cats in spread dermatophytes, the susceptibility of different epidemiological profiles and clinical syndromes associated with human dermatophytosis. hair and scales of 21 cats and 33 dogs domiciled with 25 owners with a diagnosis of dermatophytosis were collected. Among the owners, children and adults may acquire dermatophytosis from contact with dogs and cats. Most children had Tinea capitis while the adults had Tinea corporis. Between animals evaluated, 67\% (36/54) were positive to dermatophytes. Among samples evaluated, $95 \%(20 / 21)$ of feline and $49 \%$ (16/33) of the canine were positive. Symptomatic and asymptomatic evaluated cats probably transmitted the disease. On the other hand, asymptomatic dogs were probably the main spreaders of dermatophytosis to their owners. In all positive samples from cats and $94.4 \%$ of positive samples from dogs, the dermatophytes isolated were the Microsporum canis.
\end{abstract}

Key Words: adults; children; Microsporum; Tinea 


\section{INTRODUÇÃO}

A dermatofitose é uma infecção micótica de tecidos queratinizados causada por uma ou mais espécies de fungos queratinofílicos dos gêneros Microsporum, Trichophyton e Epidermophyton, os quais são denominados dermatófitos (Thomas et al., 1989; DeBoer; Moriello, 2006).

$$
\text { O principal agente da }
$$
dermatofitose em cães e gatos é o Microsporum canis, um fungo zoofílico, fortemente adaptado à pele e pelagem destes animais. $O M$. canis tem sido incriminado como causa de $82 \%$ das dermatofitoses em cães e por 97,5\% das dermatofitoses felinas (Katoh et al., 1993). Estas infecções geralmente decorrem do contato direto ou indireto com os esporos dermatofíticos presentes em ambientes contaminados, animais doentes, carreadores assintomáticos ou fômites. (Foil, 1998; DeBoer; Moriello, 2006). Adicionalmente, as dermatofitoses em animais de companhia eventualmente são causadas pelo Microsporum gypseum, um fungo geofílico, presente no solo rico em matéria orgânica, e pelo Trichophyton mentagrophytes, um fungo zoofílico e antropofílico, encontrado na pele e pelos de roedores (Bond, 2010; DeBoer; Moriello, 2006).

As dermatofitoses estão pontuadas entre as principais doenças transmitidas do animal para o ser humano (Thomas et al., 1989), e é o terceiro distúrbio tegumentar mais comum em crianças menores de 12 anos de idade e o segundo da população adulta (Pinheiro et al., 1997). Estima-se que $15 \%$ das dermatofitoses humanas são causadas por fungos zoofílicos, mormente o Microsporum canis (Larsson et al., 1997), sendo este - segundo agente etiológico mais frequente da dermatofitose humana (Londero et al., 1989; Thomas et al., 1989). diagnóstico de dermatofitose

A dermatofitose humana, causada por fungos dermatofíticos, tem sido descrita principalmente em crianças, as quais apresentam lesões geralmente no couro cabeludo (Tinea capitis), na face (Tinea faciel) ou na pele glabra do antebraço, mãos e abdome (Tinea corporis). Pessoas transplantadas, com câncer e imunocomprometidas, principalmente pela síndrome da imunodeficiência adquirida, se comportam como grupo de risco à infecção dermatofítica (Larsson et al., 1997).

Os animais carreadores assintomáticos dos esporos do $M$. canis são considerados um fator crítico na epidemiologia da doença, e $50 \%$ dos seres humanos expostos aos animais assintomáticos infectados, mormente gatos, podem se tornar infectados (Cafarchia et al., 2006).

Em um estudo realizado na universidade de Bari, na Itália, o Microsporum canis foi isolado de $36,4 \%$ dos cães e de $53,6 \%$ dos gatos que coabitavam com proprietários com diagnóstico de tinea corporis e de 14,6\% dos gatos cujos proprietários não tiveram nenhum sinal da doença (Cafarchia et al., 2006). Estes dados indicam claramente que os gatos e os cães devem ser considerados como uma fonte principal de fungos dermatofíticos para seres humanos, mesmo quando não apresentam sintomas clínicos de dermatofitose.

A prevalência do $M$. canis em infecções em humanos difere de um país para outro. $\mathrm{Na}$ Itália, esse é o dermatófito mais comumente isolado de casos de tinea capitis e tinea corporis (Cafarchia et al., 2006). No Brasil, são poucos os estudos que determinam 0 impacto de animais com dermatofitose ou carreadores assintomáticos na disseminação e contágio de seres humanos, ressaltando assim a necessidade de se avaliar a importância dos cães e gatos, domiciliados com 
proprietários com dermatofitose, como carreadores e disseminadores de fungos zoofílicos.

Devido à alta incidência da dermatofitose em cães e gatos e serem estes os principais carreadores assintomáticos de fungos zoofílicos e veiculadores da doença para o ser humano, o objetivo deste estudo foi avaliar a prevalência de infecções dermatofíticas em cães e gatos domiciliados com proprietários com dermatofitose, verificar qual o dermatófito de maior incidência nos proprietários e nos animais avaliados neste estudo e descrever o perfil epidemiológico e as síndromes clínicas encontradas nos proprietários com dermatofitose na população avaliada.

\section{MATERIAL E MÉTODOS}

$\begin{array}{cccr}\text { Foi } & \text { realizado } & \text { um } & \text { estudo } \\ \text { prospectivo, } & \text { de } & \text { coorte, } & \text { não }\end{array}$ probabilístico, em 21 gatos e 33 cães, hígidos ou com dermatofitose ou carreadores assintomáticos de esporos dermatofíticos, independente de idade, raça ou sexo, domiciliados com 25 proprietários com diagnóstico clínico e laboratorial de dermatofitose, recrutados a partir do Serviço de Dermatologia Pediátrica do Departamento de Pediatria da Faculdade de Medicina da Universidade Federal do Paraná (UFPR), do Laboratório de Micologia Médica da UFPR e do Laboratório de Micologia Mycolab de Curitiba-Paraná.

Os proprietários que procuravam atendimento médico com lesões cutâneas sob suspeita de dermatofitose eram submetidos à coleta de pelos e escamas através da esfoliação dos bordos ativos lesionais e da técnica do arrancamento, de vários locais suspeitos com uma pinça hemostática, após a limpeza local com gaze embebida em álcool a $70 \%$ e secagem ao ar, sendo a amostra submetida à cultura micológica. Quando positiva para
M. canis, eram realizadas visitas aos seus domicílios, onde se avaliavam os animais epidemiologicamente e clinicamente, distinguindo-os em sintomáticos e assintomáticos. Em seguida, eram realizadas as colheitas de pelos e escamas dos animais contactantes pelo método de Mackenzie (1963), onde a escovação dos pelos era realizada a favor e contra o sentido de sua inserção, em todo corpo do animal (face, região pré-auricular, dorso, cauda, abdome e membros), utilizando escova dental estéril.

Todas as amostras foram separadas e corretamente identificadas. Após a identificação, foram realizados repiques das amostras em placas de ágar Mycosel e acondicionados em estufa, à temperatura constante de $30^{\circ} \mathrm{C}$, sendo observado o crescimento fúngico durante três semanas. Quando necessário, microcultivo em ágar batata foi realizado visando à estimulação da formação de conídios e hifas próprias de cada espécie de fungo dermatofítico, permitindo assim sua correta identificação. Os cultivos foram realizados através de material disponível no Laboratório de Micologia do Hospital de Clínicas da Universidade Federal do Paraná.

As variáveis paramétricas foram analisadas pelo método de Quiquadrado, sendo o nível de significância adotado de $5 \% \quad(\alpha=0,05)$. Todos os cálculos foram realizados utilizando o Software estatístico GraphPad Prism ${ }^{\circledR}$ version 3.00 for Windows, San Diego Califórnia, EUA.

\section{RESULTADOS}

Foram avaliados 16 domicílios de 25 proprietários com diagnóstico confirmado de dermatofitose. Destes, três domicílios apresentavam duas pessoas com dermatofitose, um apresentava seis pessoas com dermatofitose e, nos demais apenas 

diagnóstico de dermatofitose

uma pessoa desenvolveu a doença. Dos proprietários, 14 eram crianças com idade mediana de quatro anos e meio, variando de três meses a oito anos $(P>0,05)$, sete do sexo feminino e sete do sexo masculino $(P>0,05) ; 11$ eram jovens e adultos, com idade ente $17 \mathrm{e}$ 60 anos, sendo oito mulheres e três homens $(P>0,05)$.

Das 14 crianças acometidas com a doença, duas apresentavam Tinea capitis e corporis, uma apresentava Tinea corporis e 11 apresentavam Tinea capitis $(\mathrm{P}<0,05)$.

Entre os adultos, dois apresentavam Tinea faciei, nove apresentavam Tinea corporis $(P>0,05)$ e nenhum apresentou Tinea capitis.

Foram coletados pelos e escamas de um total de 54 animais sendo 21 da espécie felina (Felis catus) e 33 da espécie canina (Canis familiaris), domiciliados com proprietários com diagnóstico de dermatofitose.

Das amostras analisadas, 67\% (36/54) apresentaram resultado positivo para 0 crescimento de fungos dermatofíticos $(P<0,001)$, entre o $3^{\circ}$ e 0 $7^{\circ}$ dia após a semeadura nos meios. Das amostras de felinos, 95\% (20/21) foram positivas $(P<0,001)$ e das amostras coletadas de cães, 51\% (16/33) foram positivas $(P<0,001)$.

Dos 33 cães avaliados, 19 eram filhotes e 14 eram adultos $(P>0,05)$. Destes, 15 eram fêmeas e 18 eram machos $(P>0,05)$, sendo um cão da raça Labrador, três da raça Pinscher e os demais não apresentavam raça definida. Todos os cães eram extradomiciliados e não possuíam contato próximo com camas e estofados, sendo que sete apresentavam sintomas da doença e 26 eram assintomáticos $(P<0,05)$.

Dos 21 gatos avaliados, 11 eram machos e 10 eram fêmeas $(P>0,05)$. Doze eram filhotes e nove eram adultos $(P>0,05)$, sendo, todos sem raça definida e, apenas um gato de pelo longo. Treze gatos eram sintomáticos e oito assintomáticos $(P>0,05)$, oito eram intradomiciliados e 13 extradomiciliados $(P>0,05)$.

Em todas as amostras positivas provenientes dos gatos analisados, 0 único dermatófito isolado foi 0 Microsporum canis $(\mathrm{P}<0,001)$. Das 16 amostras com cultura positiva provenientes dos cães, em 14 foi isolado o $M$. canis $(\mathrm{P}<0,001)$ e em duas foi isolado o Microsporum gypseum.

\section{DISCUSSÃO}

Conforme demonstrado no presente estudo, cães e gatos carreadores assintomáticos de $M$. canis ou com dermatofitose podem ser a principal fonte de contágio para seus proprietários. Dos animais analisados, domiciliados com proprietários com dermatofitose, $66,7 \%$ foram positivos para dermatófitos, o que denota que a dermatofitose humana por dermatófitos zoofílicos pode ser adquirida em ambiente intradomiciliar, a partir do contato com animais de companhia.

Paradoxalmente a literatura, esse trabalho demonstrou que tanto crianças como adultos, na mesma proporção, podem adquirir a dermatofitose por fungos zoofílicos, mormente 0 Microsporum canis, a partir do contato com cães e gatos carreadores assintomáticos ou com dermatofitose. Isso indica uma mudança no perfil social e da relação com os animais de companhia, os quais estão inseridos em ambiente familiar e ocupando os mesmos nichos do ser humano e, que a exposição a esporos dermatofíticos é o fator preponderante na disseminação da doença, já que, a pele de adultos é imunologicamente mais madura (Cafarchia et al., 2006).

Em relação às crianças e préadolescentes, observou-se que o sexo não influenciou no aparecimento da 
doença. Quanto aos jovens e adultos, a mulher foi percentualmente mais acometida, provavelmente devido ao contato mais próximo da mulher adulta aos animais de companhia do que os homens.

A síndrome clínica de maior frequência em crianças foi a Tinea capitis, fato explicado pelo contato contínuo e inadvertido da criança com o animal. Outra razão possível para a alta incidência de casos de Tinea capitis em crianças é a menor produção de sebo no couro cabeludo do que os adultos, pois, o conteúdo rico em triglicérides exerce ação fungistática contra as infecções ectotrix por Microsporum (Alchorne; Abreu, 2008).

Quanto aos adultos, as síndromes clínicas mais observadas foram a Tinea corporis e Tinea faciei. Isto provavelmente se deve ao fato de que os adultos apresentam um contato mais restrito com os animais do que as crianças, pegando os animais apenas nas mãos e, tendo pouco contato com a face e couro cabeludo.

No presente estudo, $95,2 \%$ das amostras de felinos e $48,5 \%$ das amostras de cães foram positivas, indicando ser 0 gato 0 principal disseminador de artrosporos de Microsporum canis em sua pelagem e veiculador da dermatofitose para 0 proprietário contactante.

A espécie felina é considerada a principal disseminadora de fungos zoofílicos e fonte de contágio para seres humanos e outros animais (Mignon et al., 1999; Balda et al., 2004). Isso provavelmente ocorre em função da espécie se comportar como carreadora assintomática de artroconídios dermatofíticos em índices que variam de $2,2 \%$ a $88 \%$ dos casos (Woodgyer, 1977; Quaife; Womar, 1982; Zaror et al., 1986; Gambale et al., 1993; Sparkes et al., 1994; Romano et al., 1997). Esse fato é explicado pela presença de um emulsificado lipídico na superfície da pele felina, que inibe a patogenicidade determinada pelos dermatófitos, favorecendo esse estado de carreador (Thomas et al., 1989). Sabe-se que cerca de $50 \%$ das pessoas expostas a felinos carreadores assintomáticos de artroconídios fúngicos ou portadores de infecções subclínicas, desenvolvem lesões tegumentares (Thomas et al., 1989; Mancianti et al., 2003; Cafarchia et al., 2006). Além disso, o fato do gato entrar em contato com os mesmos ambientes e ocupar os mesmos nichos dos seres humanos, em ambientes intradomiciliares favorece a transmissão.

Dos gatos analisados, tanto os assintomáticos como sintomáticos transmitiram a doença. Oito eram intradomiciliados e treze tinham acesso à rua e contato com múltiplos gatos, fato que pode favorecer a disseminação de fungos dermatofíticos e o estado de carreador assintomático na espécie.

Quanto aos cães analisados, os assintomáticos foram os principais disseminadores de dermatófitos zoofílicos e os principais veiculadores da dermatofitose aos seus proprietários. Isto pode ser explicado pelo fato do proprietário ter um contato mais contínuo e inadvertido com o cão que não apresenta lesões tegumentares, muitas vezes pegando estes no colo e mantendo-os em contato contínuo com áreas expostas e glabras do corpo e da face e com o couro cabeludo. Algumas vezes, os sintomas da dermatofitose nos animais são muito discretos, o que dificulta a identificação de animais doentes por leigos e favorece a disseminação da doença.

Em relação à faixa etária e o sexo dos animais avaliados, observouse no presente estudo que estes não influenciaram no isolamento do Microsporum canis nos cães e gatos e em sua transmissão ao ser humano, diferentemente de diversos estudos que demonstram que os animais filhotes 

diagnóstico de dermatofitose

apresentam maior capacidade de carrearem o $M$. canis em sua pelagem. (Cafarchia et al., 2006; Farias et al., 2011), pela imaturidade imune tegumentar, pela auto-higienização menos intensa e por permanecerem mais tempo aglomerados (Sympania; Baxter, 1998; Boyanowski et al., 2000; Sidrim; Rocha, 2002). A ausência de qualquer predisposição relativa ao sexo quanto à ocorrência de dermatofitose nos animais, foi também relatado por Neves et al. (2011), Palumbo et al. (2010), Balda et al. (2004) e Lewis et al. (1991). Porém, Cafarchia et al. (2004) e Baxter (1973) encontraram uma maior proporção de machos, relacionando esta maior suscetibilidade à característica de produzir maior secreção sebácea.

Em todas as amostras positivas analisadas dos gatos, e em $94,4 \%$ das amostras positivas dos cães, o dermatófito isolado foi o Microsporum canis. Esse agente também foi a principal espécie isolada em cães e gatos em outras pesquisas (Mancianti et al., 2002; Palumbo et al., 2010; Neves et al., 2011) indicando que os cães e gatos não carreiam de forma significativa esporos fúngicos de dermatófitos geofílicos, como o Microsporum gypseum, zoofílicos, como o Tricophyton mentagrophytes, ou de dermatófitos antropofílicos em sua pelagem, e o seu achado na pelagem destes é apenas acidental.

A importância dos animais de estimação com dermatofitose ou portadores sãos de dermatófitos como potenciais transmissores da doença estabelece a adoção de medidas higiênico-sanitárias para prevenção e controle. Essas medidas envolvem a participação efetiva do médico veterinário na realização de exames físico e micológico para a detecção, sobretudo, do estado de portador assintomático. Além disso, uma correta orientação aos proprietários sobre a realização de banhos com antimicóticos ativos, principalmente em animais recém chegados na propriedade, a limpeza periódica do ambiente com hipoclorito de sódio $2 \%$ e, a minimização da exposição dos animais a crianças, idosos e contactantes imunossuprimidos, bem como outros animais também representam importantes medidas profiláticas frente a novos casos desta doença em humanos (Pinheiro et al., 1997; Chermette et al., 2008).

\section{CONCLUSÃO}

O presente estudo demonstrou que a dermatofitose humana por fungos zoofílicos pode ser adquirida a partir do contato com animais de companhia, principalmente cães e gatos carreadores assintomáticos ou com dermatofitose dentro de seu ambiente domiciliar, sendo o M.canis o principal fungo disseminado pelos animais de companhia, e estes não são uma fonte de contagio e contaminação ambiental de outros fungos dermatofíticos geofílicos, zoofílicos ou antropofílicos. Tanto crianças como adultos em contato com cães e gatos doentes ou carreadores assintomáticos do Microsporum canis podem desenvolver a doença. Em relação às crianças e préadolescentes, observou-se que o sexo não influenciou o aparecimento da doença e estas apresentaram uma incidência significativa de Tinea capitis. Quando aos jovens e adultos, a mulher foi percentualmente mais acometida, sendo a Tinea corporis, a síndrome de maior ocorrência.

Através dos dados obtidos ao longo do trabalho, ressalta-se a importância da participação do Médico Veterinário no diagnóstico das dermatofitoses animais e na orientação de medidas profiláticas no sentido de preservar a saúde animal e, indiretamente, a saúde humana. 


\section{REFERÊNCIAS}

ALCHORNE, M.M.A.; ABREU, M.A.M.M. Dermatologia na pele negra. Anais Brasileiros de Dermatologia, v.83, n.1, p.7-20, 2008.

BALDA, A.C.; LARSSON, C.E.; OTSUKA, M. et al. Estudo retrospectivo de casuística das dermatofitoses em cães e gatos atendidos no Serviço de Dermatologia de Medicina Veterinária e Zootecnia da Universidade de São Paulo. Acta Scientiae Veterinariae, v.32, n.2, p.583-584, 2004

BAXTER, M. Ringworm due to Microsporum canis in cats and dogs in New Zealand. The New Zealand Veterinary Journal, v.21, n.3, p.33-37, 1973.

BOND, R. Superficial veterinary mycoses. Clinics in Dermatology, v.28, n.2, p.226-236, 2010.

BOYANOWSKI, K.J.; IHRKE, P.J.; MORIELLO, K.A. et al. Isolation of fungal flora from hair coasts of shelter cats in the Pacific coastal USA. Veterinary Dermatology, v.11, p.143-50, 2000.

CAFARCHIA, C.; ROMITO, D.; CAPELLI, G. et al. Isolation of Microsporum canis from the hair coat of pet dogs and cats belonging to owners diagnosed with $M$. canis tinea corporis.

Veterinary Dermatology, v.17, n.5, p.327-331, 2006.

CAFARCHIA, C.; ROMITO, D.; SASANELLI, M. et al. The epidemiology of canine and feline dermatophytoses in southern Italy. Mycoses, v.47, n.11/12, p.508-513, 2004.

CHERMETTE, R.; FERREIRO, L.; GUILLOT, J. Dermatophytosis in animals. Mycopathologia, v.166, n.5-6, p.385-405, 2008.

DeBOER, D. J.; MORIELLO, K. A. Cutaneous fungal infections. In: GREENE, C. E. Infectious diseases of the dog and cat. 3. ed. St. Louis, Saunders Elsevier, 2006, Cap. 58, p. 550-569.

FARIAS, M.R.; CONDAS, L.A.C.; RAMALHO, F. et al. Avaliação do estado de carreador assintomático de fungos dermatofíticos em felinos (Felis catus- linnaeus, 1793) destinados à doação em centros de controle de zoonoses e sociedades protetoras de animais. Veterinária e Zootecnia, v.18, n.2, p.306-312, 2011.

GAMBALE, W.; LARSSON, C.E.; MORITAMI, M.M. Dermatophytes and other fungy of the haircoat of cats without dermatophytosis in the city of São Paulo, Brazil. Feline Practice, v.21, n.3, p.29-33, 1993.
KATOH, T.; NISHIOKA, K.; SANO, T. A mycological study of pets as the source of human infection due to Microsporum canis. Japanese Journal of Medical Mycology, v.34, n.3, p.325-330, 1993.

LARSSON, C. E.; LUCAS, R.; GERMANO, P. M. L. Dermatofitose em cães e gatos em São Paulo: estudo da possível influência sazonal.

Anais Brasileiros de Dermatologia, v.72, n.2, 1997.

LEWIS, D.T.; FOIL, C.S.; HOSGOOD, G. Clinical and epidemiologic features of dermatophytosis in dogs and cats at Louisiana State University: 1981-1990. Veterinary

Dermatology, v.2, n.2, p.53-58, 1991.

LONDERO, A.T.; RAMOS, C.D. Agentes de dermatofitose humanas no interior do estado do Rio Grande do Sul no período de 1960 -1987.

Anais Brasileiros de Dermatologia, v.64, n.3, p.161-164, 1989.

MACKENZIE, D. W. R. "Hairbrush diagnosis" in detection and eradication of non-fluorescent scalp ringworm. British Medical Journal, v.2, n.5353, p.363-365, 1963.

MANCIANTI, F.; NARDONI, S.; CECCHI, S. et al. Dermatophytes isolated from symptomatic dogs and cats in Tuscany, Italy during a period of 15 year period. Mycopathologia, v.156, n.1, p.13-18, 2002.

MANCIANTI, F.; NARDONI, S.; CORAZZA, M. et al. Environmental detection of Microsporum canis arthrospores in the households of infected cats and dogs. Journal of Feline Medicine and Surgery, v.5, n.6, p.323-328, 2003.

MIGNON, B. R.; COIGNOUK, F.; LECLIPTEUX, $T$. et al. Histopathological pattern and humoral immune response to a crude exo-antigen and purified keratinase of Microsporum canis in symptomatic and asymptomatic infected cats. Medical Mycology, v.37, n.1, p.1-9, 1999.

NEVES, R.C.S.M.; CRUZ, F.A.C.S.; LIMA, S.R. et al. Retrospectiva das dermatofitoses em cães e gatos atendidos no Hospital Veterinário da Universidade Federal de Mato Grosso, nos anos de 2006 a 2008. Ciência Rural, v.41, n.8, 2011.

PALUMBO, M.I.P.; MACHADO, L.H.A.; PAES, A.C. et al. Estudo epidemiológico das dermatofitoses em cães e gatos atendidos no serviço de dermatologia da Faculdade de Medicina Veterinária e Zootecnia da UNESP Botucatu. Semina: Ciências Agrárias, v.31, n.2, p.459-468, 2010.

PINHEIRO, A.Q.; MOREIRA, J.L.B.; SIDRIM, J.J.C. Dermatofitose no meio urbano e a coexistência do homem com cães e gatos. 


\section{Revista da Sociedade Brasileira de Medicina}

Tropical, v.30, n.4, p.287-294, 1997.

QUAIFE, R. A.; WOMAR, S. M. Microsporum canis isolations from show cats. Veterinary Record, v.110, n.14, p.333-334, 1982.

ROMANO, C.; VALENTI, L.; BARBARA, R. Dermatophytes isolated from symptomatic stray cats. Mycoses, v.40, n.11-12, p.471-472, 1997.

SIDRIM, J.J.C.; ROCHA, M.F.G. Micologia médica à luz de autores contemporâneos. $6^{\text {a }}$ ed. Rio de Janeiro: Guanabara Koogan; 2004.

SYMPANIA, M.F.; BAXTER, M. Isolation of fungi from pelage of cats and dogs using the hairbrush technique. Mycopathologia, v.134, p.129-33, 1996.

SPARKES, A.H.; WERRETT, G.; STOKES, C.R. et al. Microsporum canis: Inapparent carriage by cats and the viability of arthrospores. Journal of Small Animal Practice, v.35, n.8, p.397-401, 1994.

THOMAS, M.L.E.; SCHEIDT, V.J.; WALKER, R.L. Inapparent carriage of $M$ canis in cats. The Compendium of Continuing Education for the Practicing Veterinarian, v.11, n.5, p.563-570, 1989.

WOODGYER, A.J. Asymptomatic carriage of dermatophytes by cats. New Zealand Veterinary Journal, v.25, n.3, p.67-69, 1977.

ZAROR, L.; FISCHMANN, O. BORGES, M. et al. The role of cats and dogs in the epidemiological cycle of Microsporum canis. Mykosen, v.29, n.4, p.185-188, 1986. 